TRABAJOS ORIGINALES

Rev Obstet Ginecol Venez. 2022; 82 (1): 33-46.

https://doi.org/10.51288/00820106

\title{
Diabetes gestacional: factores de riesgo y complicaciones perinatales*
}

\author{
Carlos Lugo León, ${ }^{1}$ (iD Naybi Bolaños, ${ }^{2}$ (iD Carla Vallejo, ${ }^{3}$ Jesús Vásquez $(†),{ }^{4}$ \\ Alexandra Rivero, ${ }^{5}$ (iD Mireya González Blanco. ${ }^{6}$
}

\section{RESUMEN}

\begin{abstract}
Objetivo: Determinar los factores de riesgo y las complicaciones perinatales en embarazadas con diabetes mellitus que acudieron a la Maternidad Concepción Palacios entre 2010 y 2015.

Métodos: Estudio retrospectivo, descriptivo y transversal que incluyó 108 pacientes diabéticas gestacionales y 108 sin diabetes. Se compararon los factores de riesgo, se evaluaron las complicaciones y sus factores de riesgo.

Resultados: Entre las diabéticas gestacionales, 76,8 \% tenían 35 o más años ( $p=0,013)$, hubo 18,5 \% primigestas ( $p=0,031), 86,9 \%$ obesas ( $p=0,000)$, $75 \%$ aumentaron más de $10 \mathrm{Kg}(p=0,000), 83,3 \%$ tenían antecedente de diabetes gestacional $(p=0,000), 31,5 \%$ antecedente familiar de hipertensión arterial crónica $(p=0,000)$ y $41 \%$ de diabetes mellitus $(p=0,001)$. Las complicaciones maternas fueron trastorno hipertensivo del embarazo $(p=0,004)$, hiperglicemias $(p=0,000)$ y estancia hospitalaria prolongada $(p=0,000)$. Las complicaciones neonatales fueron apgar bajo $(p=0,003)$, hipoglicemia $(p=0,000)$, macrosomía $(p=0,000)$ e ingreso a terapia intensiva $(p=0,001)$. Los factores de riesgo para trastorno hipertensivo del embarazo fueron 35 años o más $(p=0,0049)$, antecedentes familiares de hipertensión y diabetes $(p=0,000)$ y personales de diabetes gestacional $(p=0,0135)$. Para hiperglicemia, edad menor de 35 años $(p=0,005)$. Para complicaciones neonatales, edad menor de 35 años $(p=0,0001)$, primigestas $(p=0,0002)$, antecedente de diabetes gestacional $(p=0,000)$ y familiares de hipertensión crónica y diabetes mellitus $(p=0,0019)$.

Conclusiones: Un indice de masa corporal mayor a $25 \mathrm{~kg} / \mathrm{m}^{2}$, antecedentes personales de diabetes gestacional, familiares de diabetes e hipertensión y aumento excesivo de peso durante el embarazo, son factores de riesgo para diabetes gestacional. Las complicaciones asociadas fueron trastorno hipertensivo del embarazo e hiperglicemia.
\end{abstract}

Palabras clave: Diabetes gestacional, Factores de riesgo, Complicaciones perinatales.

\section{Gestational diabetes: risk factors and perinatal complications} SUMMARY

Objective: To determine the risk factors and perinatal complications in pregnant women with diabetes mellitus who attended the Concepción Palacios Maternity between 2010 and 2015.

Methods: Retrospective, descriptive and cross-sectional study involving 108 gestational diabetic patients and 108 without diabetes. Risk factors were compared, complications and their risk factors were assessed.

Results: Among gestational diabetics, $76.8 \%$ were 35 years of age or older (p-0.013), there were $18.5 \%$ primigestas $(p-0.031)$, 86.9\% obese (p-0.000), 75\% increased by more than $10 \mathrm{~kg}$ (p-0,000), $83.3 \%$ had a history of gestational diabetes ( $p-0.000), 31.5 \%$ family history of chronic arterial hypertension ( $p-0.000)$ and $41 \%$ diabetes mellitus ( $p$-0.001). Maternal complications were hypertensive pregnancy disorder (p-0.004), hyperglycemies $(p-0.000)$ and prolonged hospital stay $(p-0.000)$. Neonatal complications were low (p-0.003), hypoglycemia ( $p-0.000)$, macrosomia (p-0.000) and admission to intensive therapy ( $p-0.001)$. Risk factors for hypertensive pregnancy disorder were 35 years or more ( $p-0.0049)$, a family history of hypertension and diabetes ( $p-0.000)$ and personal history of gestational diabetes (p-0.0135). For hyperglycemia, age 35 years lower than 35 years $(p-0.005)$. For neonatal complications, age less than 35 years ( $p-0.0001)$, primigestas ( $p-0.0002)$, history of gestational diabetes $(p-0.000)$ and relatives of chronic hypertension and diabetes mellitus (p-0.0019).

Conclusions: A body mass index greater than $25 \mathrm{~kg} / \mathrm{m}^{2}$, a personal history of gestational diabetes, family members of diabetes and hypertension, and excessive weight gain during pregnancy are risk factors for gestational diabetes. Associated complications were hypertensive pregnancy disorder and hyperglycemia.

Keywords: Gestational diabetes, Risk factors, Perinatal complications.

${ }^{1}$ Director Nacional Sociedad Venezolana de Ultrasonido en medicina, Miembro titular SOGV. Especialista en Medicina Materno Fetal. Obstetra ginecólogo. ${ }^{2}$ Especialista en Medicina Materno Fetal. Obstetra ginecólogo. ${ }^{3}$ Especialista en Medicina Materno Fetal. Obstetra ginecólogo. ${ }^{4}$ Obstetra ginecólogo. ${ }^{5}$ Miembro titular SOGV. Especialista en Medicina Materno Fetal. Obstetra ginecólogo. ${ }^{6}$ Miembro titular SOGV. Directora/Editora de la revista SOGV. Obstetra ginecólogo. *Trabajo realizado para optar el título académico en Especialista en Medicina Materno Fetal de la Universidad Central de Venezuela. Correo para correspondencia: lugomaternofetal@gmail.com

Forma de citar este artículo: Lugo León C, Bolaños $\mathrm{N}$, Vallejo C, Vasquez J, Rivero A, Gonzalez-Blanco M. Diabetes gestacional: factores de riesgo y complicaciones perinatales. Rev Obstet Ginecol Venez. 2022; 82(1): 33-46. https://doi.org/10.51288/00820106

\section{INTRODUCCIÓN}

La diabetes mellitus (DM) constituye la alteración metabólica que más frecuentemente se asocia al embarazo, con una incidencia de $1 \%$; se sabe que la DM tipo 1 y tipo 2 afectan el pronóstico de la madre $\mathrm{y}$ el de su futuro hijo, con un incremento del riesgo 
de abortos, malformaciones y otras complicaciones perinatales (1).

Durante muchos años se definió a la diabetes gestacional como cualquier grado de intolerancia a la glucosa observado por primera vez durante el embarazo, independientemente de si la causa precedía o persistía después de la gestación (1); dicha definición facilitó una estrategia de detección y clasificación de la patología, pero era limitada a causa de su imprecisión. La actual epidemia de obesidad en mujeres en edad fértil ha conducido al aumento de pacientes embarazadas con diabetes no diagnosticada.

Debido al número de diabéticas embarazadas no diagnosticadas, es fundamental el cribado de dicha patología en el comienzo del control prenatal, logrando de esta manera iniciar un reconocimiento de la patología tempranamente, factores de riesgo, manejo y tratamiento. Toda paciente que sea diagnosticada por encima de la semana 20 de gestación debe catalogarse como diabética gestacional, mientras que aquellas evidenciadas antes de este periodo se corresponderán con diabetes tipo 2 (2).

La prevalencia de diabetes gestacional varía entre $1 \%$ y $14 \%$ de todos los embarazos, en función de las características genéticas, el entorno de la población objeto de estudio, la detección y los métodos diagnósticos así como la prevalencia de diabetes mellitus tipo 2 (DM-2). Para el año 2013, se estimó que la prevalencia global de embarazadas con edades comprendidas entre los 20 años y 49 años era de $16,9 \%$, lo cual correspondía con 21,4 millones de mujeres aproximadamente. Más de $90 \%$ de los casos de hiperglicemia en el embarazo ocurre en países de medianos y bajos ingresos, la prevalencia más alta fue en el oriente medio y norte de África $(22,3 \%)$ en donde los Emiratos Árabes presentan la mayor prevalencia con $37 \%$, seguido de Noruega con $31 \%$ y la India con $28 \%$; en comparación con regiones de
América del Norte y el Caribe 10,4 \% (3, 4). Estudios poblacionales en Venezuela estiman la prevalencia de dicha enfermedad entre $2 \%$ y $3 \%$ aproximadamente (5).

Los factores de riesgo comúnmente asociados a la aparición de diabetes gestacional no han variado considerablemente con el pasar de los años, destacan el sobrepeso y la obesidad por ser modificables; edad mayor a 35 años, antecedentes familiares de DM-2 o personales de diabetes gestacional (DG), síndrome de ovario poliquísticos, macrosomía y muerte fetal inexplicada en el tercer trimestre, entre otros $(3,5)$.

Entre las 24 y 28 semanas de embarazo debe realizarse la curva de glicemia con carga oral de 75 gramos de glucosa, basado en las recomendaciones de la Asociación Internacional de Diabetes y Grupos de Estudio en el Embarazo (IADPSG), quienes definen tres puntos de corte para la evaluación (ayuno, 1 hora y 2 horas), considerando una paciente diabética gestacional si presenta valores iguales o mayores a $92 \mathrm{mg} / \mathrm{dL}, 180 \mathrm{mg} / \mathrm{dL}, 153 \mathrm{mg} / \mathrm{dL}$ respectivamente $(2,6)$. El estudio realizado sobre la hiperglicemia y los resultados adversos del embarazo (HAPO) (7) demostró que el riesgo de resultados adversos maternos, fetales y neonatales aumentó continuamente en función de la glicemia materna entre las 24 y 28 semanas de gestación, incluso dentro de los rangos considerados como normales, sin haber un umbral de riesgo para la mayoría de las complicaciones $(2,7)$.

En aproximadamente, entre $80 \%$ a $90 \%$ de las pacientes con DG, el tratamiento solo dependerá de modificar la dieta e iniciar actividad física. (2)

La DG no se encuentra dentro de las principales causas de mortalidad materna sin embargo, complicaciones tales como preeclampsia, infección, distocias del trabajo de parto y abortos están ligadas a las madres diabéticas; del mismo modo la diabetes gestacional 
puede contribuir con patologías y alteraciones en el producto de la concepción teniendo un impacto significativo en los costos e infraestructura hospitalaria $(3,8)$.

Estudios observacionales han demostrado que el riesgo de malformaciones fetales está relacionado principalmente con el mal control glicémico en las primeras $6-8$ semanas de gestación, motivo por el cual, la optimización del control debería iniciarse antes de la concepción $\mathrm{y}$, por tanto, la gestación debería estar planificada. Sin embargo, alrededor de unas dos terceras partes de las mujeres diabéticas no realizan planificación de la gestación (1).

A nivel mundial, la diabetes mellitus es una complicación frecuente durante el embarazo, los criterios diagnósticos se han modificado con el pasar de los años para lograr un manejo adecuado tan temprano como sea posible, buscando determinar y disminuir las complicaciones tanto maternas como fetales.

Sobre la base de lo anteriormente expuesto se planteó investigar los factores de riesgo para desarrollar diabetes gestacional así como las complicaciones maternas y perinatales más frecuentes en pacientes con edad gestacional comprendida entre 20 y 41 semanas de embarazo, que ingresaron en el Servicio de Medicina Materno Fetal de la Maternidad Concepción Palacios, entre enero 2010 y diciembre 2015.

Teniendo presente que la diabetes mellitus representa una de las primeras causas de patologías médicas, con complicaciones maternas y fetales durante el embarazo que se encuentran en relación directa con los niveles de glicemia presentes en la madre antes y durante la gestación, es importante determinar los principales factores de riesgo en las pacientes que cursen con diabetes gestacional, logrando así establecer un plan de trabajo y directrices orientadas en el diagnóstico y manejo adecuado para incidir directamente sobre la morbimortalidad materna y perinatal.
La Unidad de Medicina Materno Fetal en la Maternidad Concepción Palacios no cuenta con estudios propios que evalúen los factores de riesgo y complicaciones asociadas a diabetes gestacional, logrando de esta manera la categorización, evolución y manejo de las pacientes ante dicha patología, es por ello que este trabajo puede orientar en la práctica médica diaria, siendo pionero a nivel institucional. De igual forma, se espera que la investigación sirva de referencia para futuras investigaciones, logrando implementar líneas de investigación destinadas a mejorar la atención médica de las pacientes.

\section{MÉTODOS}

Se realizó un estudio retrospectivo, transversal, de casos y controles. La población estuvo representada por 4200 historias médicas de pacientes que acudieron al Servicio de Medicina Materno Fetal de la Maternidad Concepción Palacios para atención obstétrica en un año. La muestra se calculó usando la fórmula para población infinita, considerando un nivel de confianza de $95 \%$ y una precisión de $5 \%$, con una proporción de $7 \%$ (prevalencia promedio de diabetes gestacional en la literatura), con una pérdida de seguimiento del $10 \%$, se obtuvo una muestra de 108 pacientes. Por lo tanto, se seleccionó una muestra aleatoria de 108 pacientes del grupo de las 490 pacientes con diagnóstico de diabetes gestacional para el grupo experimental y 108 pacientes del resto de la población, sin diagnóstico de diabetes gestacional, para el grupo control. Se incluyeron embarazadas con edad gestacional comprendida entre 20 -41 semanas, con diagnóstico de diabetes gestacional en el grupo experimental, y no diabéticas en el grupo control. Se excluyeron aquellas con anormalidades cromosómicas o estructurales, patologías inmunológicas, diabetes mellitus (pregestacional) y las pacientes cuyas historias no estaban localizables o los datos en la misma eran incompletos. 
Se realizó una revisión aleatoria de las historias clínicas. Se compararon los eventuales factores de riesgo entre ambos grupos. Posteriormente, las pacientes del grupo con diagnóstico de diabetes gestacional que presentaron complicaciones se compararon con aquellas que no las presentaron para determinar los factores de riesgo de complicaciones.

Los resultados fueron elaborados en atención a las variables de investigación de las mujeres que cumplieron con los criterios de inclusión, calculando indicadores descriptivos de acuerdo al tipo de variable estadística, inferencialmente, se calcularon contrastes de hipótesis con el estadístico exacto de Fisher a un nivel de confianza del $95 \%$, se consideraron diferencias estadísticamente significativas cuando $\mathrm{p}<0,05$, posteriormente se calculó el Odds Ratio para determinar los factores de riesgo.

\section{RESULTADOS}

En la tabla 1 se presenta la distribución comparativa según los factores de riesgo conocidos para diabetes gestacional. En el grupo de pacientes diabéticas, 83 mujeres $(76,8 \%)$ tenían 35 o más años, en el grupo de las no diabéticas, 67 (62\%) pertenecían a este grupo de edad $(\mathrm{p}=0,013)$. En relación con la paridad, 20 (18,5\% de las diabéticas) eran primigestas mientras

Tabla 1. Distribución de pacientes según los factores de riesgo para diabetes gestacional

\begin{tabular}{|c|c|c|c|c|c|c|}
\hline \multirow{3}{*}{$\begin{array}{l}\text { Factor de riesgo } \\
\text { Edad }\end{array}$} & \multicolumn{2}{|c|}{$\begin{array}{c}\text { Pacientes con } \\
\text { diabetes gestacional }\end{array}$} & \multicolumn{2}{|c|}{$\begin{array}{c}\text { Pacientes sin } \\
\text { diabetes gestacional }\end{array}$} & \multirow{3}{*}{$\begin{array}{c}\mathrm{p} \\
0,013\end{array}$} & \multirow{3}{*}{$\begin{array}{c}\begin{array}{c}\text { Riesgo } \\
\text { relativo }\end{array} \\
0,4\end{array}$} \\
\hline & $\mathrm{n}$ & $\%$ & $\mathrm{n}$ & $\%$ & & \\
\hline & & & & & & \\
\hline Inferior a 35 años & 25 & 23,2 & 41 & 38 & & \\
\hline 35 o más años & 83 & 76,8 & 67 & 62 & & \\
\hline Paridad & & & & & 0,031 & \\
\hline Primigestas & 20 & 18,5 & 33 & 30,5 & & 1,0 \\
\hline 2 a 4 & 63 & 58,3 & 58 & 53,7 & & 1,3 \\
\hline 5 o más & 25 & 23,1 & 17 & 15,7 & & 1,5 \\
\hline Recién nacidos macrosómicos previos & 3 & 1,8 & 1 & 0,9 & 0,621 & 3,0 \\
\hline Abortos recurrentes & 7 & 6,5 & 5 & 4,6 & 0,768 & 1,4 \\
\hline Óbitos & 3 & 2,8 & 1 & 0,9 & 0,621 & 3,0 \\
\hline Peso materno & & & & & 0,000 & 14,0 \\
\hline Normal & 7 & 6,4 & 70 & 64,8 & & \\
\hline Sobrepeso & 7 & 6,4 & 3 & 2,7 & & \\
\hline Obesidad & 46 & 42,5 & 12 & 11,1 & & \\
\hline Obesidad severa & 48 & 44,4 & 23 & 21,2 & & \\
\hline Aumento excesivo de peso en el embarazo & 81 & 75 & 45 & 41,7 & 0,000 & 4,2 \\
\hline \multicolumn{7}{|l|}{ Antecedentes personales } \\
\hline Diabetes gestacional & 90 & 83,3 & 4 & 3,7 & 0,000 & 130,0 \\
\hline Enfermedades tiroideas & 3 & 2,8 & 1 & 0,9 & 0,621 & 3,0 \\
\hline Hipertensión en el embarazos & 2 & 1,8 & 5 & 8,6 & 0,445 & \\
\hline \multicolumn{7}{|l|}{ Antecedentes familiares } \\
\hline Hipertensión arterial crónica & 34 & 31,5 & 62 & 57,9 & 0,000 & \\
\hline Diabetes mellitus tipo 2 & 41 & 38 & 19 & 17,8 & 0,001 & 2,8 \\
\hline \multicolumn{7}{|l|}{ Condiciones socioeconómicas } \\
\hline Bajo nivel educativo & 9 & 8,3 & 14 & 12,9 & 0,378 & \\
\hline Bajo ingreso económico & 84 & 77,8 & 84 & 77,8 & 1,000 & \\
\hline
\end{tabular}


entre las no diabéticas había 33 primigestas (30,5\%) $(\mathrm{p}=0,031)$. Entre las diabéticas, $45,4 \%$ tenían 37 semanas o más y en el grupo control el porcentaje de mujeres que presentaban más de 37 semanas fue 34,3\%.

Respecto al peso materno, entre las diabéticas, el índice de masa corporal (IMC) osciló entre 20 y $47 \mathrm{~kg} / \mathrm{m}^{2}$, con una media de $31,1 \pm 5,5 \mathrm{~kg} / \mathrm{m}^{2}$, mientras que la mediana fue de $30 \mathrm{~kg} / \mathrm{m}^{2}$. Entre las no diabéticas, el rango de IMC fue 20 y $50 \mathrm{~kg} / \mathrm{m}^{2}$, una media de $26,4537 \pm 6,6591 \mathrm{~kg} / \mathrm{m}^{2}$, y la mediana fue de $24 \mathrm{~kg} / \mathrm{m}^{2}$. Noventa y cuatro diabéticas $(86,9 \%)$ eran obesas y, de ellas, 48 tenían obesidad severa. Entre las no diabéticas, 70 tenían peso normal $(64,8 \%)$ $(\mathrm{p}=0,000) \quad(\mathrm{RR}: \quad 14,0 ; \mathrm{p}<0,05)$. Hubo aumento excesivo de peso (más de $10 \mathrm{Kg}$ ) en 81 diabéticas ( $75 \%$ ) y en 45 no diabéticas (41,7\%), en el grupo de no diabéticas, $58,7 \%$ adicional ganaron entre 5 y $10 \mathrm{Kg}$ $(p=0,000)(R R: 4,2 ; p<0,05)$.
Se encontró antecedente de diabetes gestacional en 90 diabéticas $(83,3 \%)$ y en 4 no diabéticas $(3,7 \%)$ $(p=0,000)(R R: 130 ; p<0,05)$. Entre las diabéticas, 34 $(31,5 \%)$ tenían antecedente familiar de hipertensión arterial crónica y 41 de diabetes mellitus (38 \%). En las no diabéticas, esos antecedentes se presentaron en $62(57,9 \%)$ y $19(17,8 \%)$ casos, respectivamente $(\mathrm{p}=0,000$ y $\mathrm{p}=0,001)$. No se encontró relación entre el diagnóstico de diabetes gestacional y el antecedente de recién nacidos macrosómicos $(\mathrm{p}=0,621)$, abortos recurrentes $(p=0,768)$, óbitos fetales $(p=0,621)$, enfermedades tiroideas $(p=0,621)$, hipertensión en embarazos previos $(\mathrm{p}=0,445)$, nivel educativo $(\mathrm{p}=0,378)$ o ingreso económico $(\mathrm{p}=1,000)$.

En relación con las complicaciones maternas, en la tabla 2 se puede observar que entre las pacientes con diabetes gestacional se presentaron 29 casos $(26,9 \%)$ de trastorno hipertensivo del embarazo (THE), 47

Tabla 2. Distribución comparativa de las complicaciones maternas entre pacientes con y sin diabetes gestacional

\begin{tabular}{|c|c|c|c|c|c|}
\hline \multirow[t]{2}{*}{ Complicaciones } & \multicolumn{2}{|c|}{$\begin{array}{c}\text { Pacientes con } \\
\text { diabetes gestacional }\end{array}$} & \multicolumn{2}{|c|}{$\begin{array}{c}\text { Pacientes sin } \\
\text { diabetes gestacional }\end{array}$} & \multirow{2}{*}{$\mathrm{p}$} \\
\hline & $\mathrm{n}$ & $\%$ & $\mathrm{n}$ & $\%$ & \\
\hline \multicolumn{6}{|l|}{ Durante el embarazo (Obstétricas) } \\
\hline $\mathrm{RPM}^{*}$ & 17 & 15,7 & 9 & 8,3 & 1,422 \\
\hline Parto pretérmino & 6 & 5,6 & 4 & 3,7 & 0,748 \\
\hline Trastorno hipertensivo del embarazo & 29 & 26,9 & 16 & 14,8 & 0,004 \\
\hline \multicolumn{6}{|l|}{ Durante el embarazo (Médicas) } \\
\hline Cetoacidosis diabética & 2 & 1,8 & 0 & 0 & 0,497 \\
\hline Infecciones urinarias recurrentes & 5 & 4,6 & 10 & 9,3 & 0,284 \\
\hline Hiperglicemias & 47 & 43,5 & 0 & 0 & 0,000 \\
\hline Hipoglicemias & 4 & 3,7 & 0 & 0 & 0,122 \\
\hline \multicolumn{6}{|l|}{ Durante el parto } \\
\hline Vía del parto & & & & & 0,109 \\
\hline Vaginal espontáneo & 20 & 18,6 & 31 & 28,7 & \\
\hline Cesárea & 88 & 81,4 & 77 & 70,3 & \\
\hline Distocia de hombros & 3 & 2,7 & 2 & 1,8 & 0,445 \\
\hline Laceraciones del canal del parto & 10 & 9,3 & 6 & 5,6 & 0,436 \\
\hline \multicolumn{6}{|l|}{ Después del embarazo } \\
\hline Estancia hospitalaria $\geq 8 \mathrm{~d}$ & 48 & 44,4 & 0 & 0 & 0,000 \\
\hline
\end{tabular}

*RPM: ruptura prematura de membranas 
Tabla 3. Distribución comparativa de las complicaciones neonatales entre recién nacidos de madres con y sin diabetes gestacional

\begin{tabular}{lccccc}
\hline \multirow{2}{*}{ Complicaciones } & \multicolumn{3}{c}{ Pacientes con } & \multicolumn{2}{c}{ Pacientes sin } \\
& diabetes gestacional & \multicolumn{2}{c}{ diabetes gestacional } & $\mathrm{p}$ \\
\cline { 2 - 5 } & $\mathrm{n}$ & $\%$ & $\mathrm{n}$ & $\%$ & \\
\hline Durante el embarazo (obstétricas) & 3 & 2,7 & 2 & 1,8 & 1,000 \\
$\quad$ Defectos congénitos & 3 & 2,7 & 1 & 0,9 & 0,621 \\
$\quad$ Óbito Fetal & & & & & \\
$\quad$ Durante el parto & 3 & 2,7 & 2 & 1,8 & 0,480 \\
$\quad$ Distocia de hombro & 3 & 2,7 & 2 & 1,8 & 0,312 \\
$\quad$ Trauma durante el parto & & & & & \\
$\quad$ Después del nacimiento & 10 & 9,2 & 2 & 1,8 & 0,003 \\
$\quad$ Apgar menor a 5 en el primer minuto & 27 & 25 & 1 & 0,9 & 0,000 \\
$\quad$ Hipoglicemia & 13 & 12 & 0 & 0 & 0,000 \\
Macrosomía neonatal & 49 & 45,4 & 12 & 11,7 & 0,008 \\
$\quad$ Ingreso a UTI* & & & &
\end{tabular}

*Unidad de terapia intensiva

pacientes con hiperglicemias $(43,5 \%)$ y $48(44,4 \%)$ pacientes quienes requirieron estancia hospitalaria prolongada, es decir, 8 días o más. En el grupo que no presentó diabetes gestacional se diagnosticó THE en 16 casos $(14,8 \%)$, ninguna hiperglicemia y ninguna requirió ingreso por 8 días o más; $(p=0,004, p=0,000$ $\mathrm{y} p=0,000$, respectivamente). No se observó relación entre la diabetes gestacional y el diagnóstico de ruptura prematura de membranas $(\mathrm{RPM})(\mathrm{p}=1,422)$, parto pretérmino $(\mathrm{p}=0,748)$, cetoacidosis diabética $(\mathrm{p}=0,497)$, infección urinaria recurrente $(\mathrm{p}=0,284)$, hipoglicemias $(\mathrm{p}=0,122)$, necesidad de cesárea $(\mathrm{p}=0,109)$, distocia de hombros $(\mathrm{p}=0,445)$, ni laceraciones del canal del parto $(\mathrm{p}=0,436)$.

En la tabla 3 se observan las complicaciones neonatales. Se encontró un apgar menor a 5 en el primer minuto en $10(9,2 \%)$ de los recién nacidos de madres diabéticas gestacionales y en $2(1,8 \%)$ de las que no tenían el diagnóstico $(\mathrm{p}=0,003)$. Asimismo, en el primer grupo hubo 27 neonatos con hipoglicemia (25\%), 13 con macrosomía (12\%) y 49 que ameritaron ingresar a la unidad de terapia intensiva neonatal (UTIN) $(45,4 \%)$. En el segundo grupo, hubo un solo caso de hipoglicemia $(0,9 \%)$, ninguna macrosomía y 12 ameritaron ingreso a UTIN (11,7 \%) ( $p=0,000, p=0,000$ y $p=0,001$, respectivamente). No hubo relación entre la diabetes gestacional y la presencia de defectos congénitos $(p=1,000)$, óbito fetal $(p=0,621)$, distocia de hombros $(\mathrm{p}=0,445)$ ni trauma durante el parto $(\mathrm{p}=0,279)$.

Para establecer los factores de riesgo para complicaciones en el grupo de pacientes con diabetes gestacional, se evaluaron aquellas con diagnóstico de THE, hiperglicemia y prolongada estancia hospitalaria, por ser las únicas complicaciones relacionadas con la patología en estudio $(\mathrm{p}=0,004, \mathrm{p}=0,000, \mathrm{p}=0,000)$.

En el caso de los THE, se observa en la tabla 4 que 24 de las gestantes complicadas $(82,8 \%)$ y 59 de las no complicadas $(74,7 \%)$, tenían 35 años o más $(\mathrm{p}=0,0049)$. Once del primer grupo $(37,9 \%)$ y 52 del segundo (65,8\%), tenían 2 a 4 embarazos previos $(p=0,0012)$. Hubo 3 recién nacidos $(R N)$ macrosómicos previos entre las no complicadas (3,8\%) y ninguno entre las que presentaron THE. Entre las diabéticas gestacionales que presentaron THE, hubo $29(100 \%)$ con diabetes gestacional en el embarazo previo, $2(0,5 \%)$ con hipertensión en embarazo previo y $2(0,5 \%)$ con patologías tiroideas; 20 (69\%) con 
Tabla 4. Distribución según los factores de riesgo para complicaciones maternas de la diabetes gestacional: Trastorno hipertensivo del embarazo

\begin{tabular}{|c|c|c|c|c|c|c|}
\hline \multirow{3}{*}{ Factor de riesgo } & \multicolumn{4}{|c|}{ Pacientes con diabetes gestacional } & \multirow{3}{*}{$\mathrm{p}$} & \multirow{3}{*}{$\begin{array}{l}\text { Riesgo } \\
\text { relativo }\end{array}$} \\
\hline & \multicolumn{2}{|c|}{$\begin{array}{c}\text { Complicadas } \\
\text { con THE* } n=29\end{array}$} & \multicolumn{2}{|c|}{$\begin{array}{l}\text { No complicadas } \\
\text { con THE* } \mathrm{n}=79\end{array}$} & & \\
\hline & $\mathrm{n}$ & $\%$ & $\mathrm{n}$ & $\%$ & & \\
\hline Edad & & & & & 0,0049 & \\
\hline Inferior a 35 años & 5 & 17,2 & 20 & 25,3 & & 0,25 \\
\hline 35 o más años & 24 & 82,8 & 59 & 74,7 & & 0,41 \\
\hline Paridad & & & & & 0,0012 & \\
\hline Primigestas & 8 & 27,6 & 12 & 15,2 & & 0,67 \\
\hline 2 a 4 & 11 & 37,9 & 52 & 65,8 & & 0,21 \\
\hline 5 o más & 10 & 34,5 & 15 & 19 & & 0,67 \\
\hline Recién nacidos macrosómicos previos & 0 & - & 3 & 3,8 & 0,0001 & 0,00 \\
\hline Abortos recurrentes & 3 & 10,3 & 4 & 5,1 & 0,5673 & 0,75 \\
\hline Óbitos & 1 & 3,4 & 2 & 2,5 & 0,4590 & 0,50 \\
\hline Peso materno & & & & & 0,7438 & \\
\hline Normal & 3 & 10,3 & 4 & 5,1 & & 0,75 \\
\hline Sobrepeso & 6 & 20,7 & 1 & 1,3 & & 6,00 \\
\hline Obesidad & 14 & 48,3 & 32 & 40,5 & & 0,44 \\
\hline Obesidad severa & 6 & 27,6 & 42 & 53,2 & & 0,14 \\
\hline Aumento excesivo de peso en el embarazo & 22 & 75,9 & 59 & 74,7 & 0,1632 & 0,37 \\
\hline Antecedentes personales & & & & & 0,0135 & \\
\hline Diabetes gestacional & 29 & 100 & 61 & 77,2 & & 0,48 \\
\hline Enfermedades tiroideas & 2 & 0,5 & 1 & 1,3 & & 2,00 \\
\hline Hipertensión en el embarazos & 2 & 0,5 & 0 & - & & \\
\hline Antecedentes familiares & & & & & 0,0000 & \\
\hline Hipertensión arterial crónica & 20 & 69,0 & 14 & 17,7 & & 10,32 \\
\hline Diabetes mellitus tipo 2 & 28 & 96,6 & 13 & 16,5 & & 14,1 \\
\hline Condiciones socioeconómicas & & & & & 0,1030 & \\
\hline Bajo nivel educativo & 3 & 10,3 & 6 & 7,6 & & 0,50 \\
\hline Bajo ingreso económico & 29 & 100 & 55 & 69,6 & & 0,53 \\
\hline
\end{tabular}

*THE: trastorno hipertensivo del embarazo

antecedentes familiares de hipertensión arterial y $28(96,6 \%)$ con antecedentes familiares de diabetes mellitus tipo 2. Las que no se complicaron con THE, $61(77,2 \%)$ tenían antecedentes personales de diabetes gestacional, ninguna tenía antecedentes de hipertensión y una $(1,3 \%)$ tenía patología tiroidea; en 14 casos $(17,7 \%)$ había hipertensión arterial en la familia y en $13(16,5 \%)$ había diabetes mellitus tipo $2(p=0,0135$ para antecedentes personales y $p=0,000$ para antecedentes familiares).

En relación con el desarrollo de hiperglicemia, la tabla 5 muestra que se asoció a edad materna: 20 (42,6\%) complicadas y $5(8,2 \%)$ no complicadas eran menores de 35 años $(\mathrm{p}=0,0052)$, a recién nacidos macrosómicos previos: dos $(4,3 \%)$ entre las que presentaron hiperglicemia y $1(1,6 \%)$ entre las que no $(\mathrm{p}=0,0014)$. No hubo óbitos entre las complicadas y hubo 3 (4,9\%) entre las no complicadas $(p=0,000)$. Entre las que presentaron hiperglicemia, $32(68,1 \%)$ ganaron más de 10 kilos de peso en el embarazo. Entre las que no presentaron hiperglicemia, $49(80,3 \%)$ presentaron aumento excesivo de peso $(\mathrm{p}=0,000)$. Entre las diabéticas gestacionales que presentaron hiperglicemia, $6(12,8 \%)$ tenían bajo nivel educativoy47(100\%)teníanbajoingresoeconómico.Enel grupo de las que no presentaron hiperglicemia, 3 (4,9\%) 
Tabla 5. Distribución según los factores de riesgo para complicaciones maternas de la diabetes gestacional: hiperglicemia

\begin{tabular}{|c|c|c|c|c|c|c|}
\hline \multirow{3}{*}{ Factor de riesgo } & \multicolumn{4}{|c|}{ Pacientes con diabetes gestacional } & \multirow{3}{*}{$\mathrm{p}$} & \multirow{3}{*}{$\begin{array}{l}\text { Riesgo } \\
\text { relative }\end{array}$} \\
\hline & \multicolumn{2}{|c|}{$\begin{array}{c}\text { Complicadas con } \\
\text { hiperglicemia } n=47\end{array}$} & \multicolumn{2}{|c|}{$\begin{array}{l}\text { No complicadas con } \\
\text { hiperglicemia } n=61\end{array}$} & & \\
\hline & $\mathrm{n}$ & $\%$ & $\mathrm{n}$ & $\%$ & & \\
\hline Edad & & & & & 0,0052 & \\
\hline Inferior a 35 años & 20 & 42,6 & 5 & 8,2 & & 4,00 \\
\hline 35 o más años & 27 & 57,4 & 56 & 91,8 & & 0,48 \\
\hline Paridad & & & & & 0,8143 & \\
\hline Primigestas & 12 & 25,5 & 8 & 13,1 & & 1,50 \\
\hline 2 a 4 & 20 & 42,6 & 43 & 70,5 & & 0,47 \\
\hline 5 o más & 15 & 31,9 & 10 & 16,4 & & 1,50 \\
\hline Recién nacidos macrosómicos previos & 2 & 4,3 & 1 & 1,6 & 0,0014 & 2,00 \\
\hline Abortos recurrentes & 2 & 4,3 & 5 & 8,2 & 0,3808 & 0,40 \\
\hline Óbitos & 0 & - & 3 & 4,9 & 0,0000 & 0,00 \\
\hline Peso materno & & & & & 0,6230 & \\
\hline Normal & 5 & 10,6 & 2 & 3,3 & & 0,97 \\
\hline Sobrepeso & 4 & 8,5 & 3 & 4,9 & & 2,78 \\
\hline Obesidad & 22 & 46,8 & 24 & 39,4 & & 2,47 \\
\hline Obesidad severa & 16 & 34 & 32 & 52,5 & & 5,68 \\
\hline Aumento excesivo en el embarazo & 32 & 68,1 & 49 & 80,3 & 0,0000 & 0,65 \\
\hline Antecedentes personales & & & & & 0,9870 & \\
\hline Diabetes gestacional & 47 & 100 & 43 & 70,5 & & 1,09 \\
\hline Enfermedades tiroideas & 1 & 2,1 & 2 & 3,3 & & 0,50 \\
\hline Hipertensión en el embarazos & 0 & - & 2 & 3,3 & & 0,00 \\
\hline Antecedentes familiares & & & & & 0,0907 & \\
\hline Hipertensión arterial crónica & 10 & 21,3 & 24 & 39,3 & & 0,42 \\
\hline Diabetes mellitus tipo 2 & 20 & 42,6 & 21 & 34,4 & & 0,95 \\
\hline Condiciones socioeconómicas & & & & & 0,0297 & \\
\hline Bajo nivel educativo & 6 & 12,8 & 3 & 4,9 & & 2,00 \\
\hline Bajo ingreso económico & 47 & 100 & 37 & 60,7 & & 1,27 \\
\hline
\end{tabular}

tenían bajo nivel educativo y 37 (60,7 \%) bajo ingreso económico $(\mathrm{p}=0,0297)$. La hiperglicemia no se asoció a la paridad $(\mathrm{p}=0,8143)$, abortos recurrente $(\mathrm{p}=0,3808)$, peso materno $(\mathrm{p}=0,6230)$, antecedentes personales $(\mathrm{p}=0,9870)$, ni familiares $(\mathrm{p}=0,0907)$.

En la tabla 6 se observa que entre los factores de riesgo evaluados, solo tener recién nacidos macrosómicos previos, se asoció a no requerir estancia hospitalaria prolongada; entre aquellas cuyo ingreso duró más de 8 días, ninguna tuvo recién nacidos macrosómicos previos y entre las que estuvieron ingresadas por menos de 8 días, hubo tres $(5 \%)$ con el antecedente $(\mathrm{p}=0,0021)$.
Hubo 52 neonatos complicados. En la tabla 7 se presentan los factores de riesgo que podrían asociarse a complicaciones en los recién nacidos de madres con diabetes gestacional. Entre los no complicados, hubo 22 madres menores de 35 años (42,3\%) y solo 3 (5,3\%) entre los no complicados $(\mathrm{p}=0,0001)$, así mismo, en el primer grupo $12(23,1 \%)$ eran primigestas y en el segundo grupo solo $8(13,4 \%)(p=0,0001)$; los 52 complicados (10\%) eran hijos de madres con diabetes gestacional previa $(p=0,000), 24(46,2 \%)$ tenían antecedente familiar de hipertensión crónica y $30(57,7 \%)$ de diabetes mellitus tipo $2(p=0,0001)$. No hubo asociación con los demás factores evaluados. 
Tabla 6. Distribución según los factores de riesgo para complicaciones maternas de la diabetes gestacional: estancia hospitalaria prolongada

\begin{tabular}{|c|c|c|c|c|c|c|}
\hline \multirow{3}{*}{ Factor de riesgo } & \multicolumn{4}{|c|}{ Pacientes con diabetes gestacional } & \multirow{3}{*}{$\mathrm{p}$} & \multirow{3}{*}{$\begin{array}{l}\text { Riesgo } \\
\text { relativo }\end{array}$} \\
\hline & \multicolumn{2}{|c|}{$\begin{array}{c}\text { Estancia hospitalaria } \\
\text { prolongada } \\
n=48\end{array}$} & \multicolumn{2}{|c|}{$\begin{array}{c}\text { Estancia hospitalaria } \\
\text { no prolongada } \\
n=60\end{array}$} & & \\
\hline & $\mathrm{n}$ & $\%$ & $\mathrm{n}$ & $\%$ & & \\
\hline Edad & & & & & 0,2431 & \\
\hline Inferior a 35 años & 20 & 41,7 & 5 & 8,3 & & 4,00 \\
\hline 35 o más años & 28 & 58,3 & 55 & 91,7 & & 0,51 \\
\hline Paridad & & & & & 0,5927 & \\
\hline Primigestas & 10 & 20,8 & 10 & 16,7 & & 1,00 \\
\hline 2 a 4 & 28 & 58,3 & 35 & 58,3 & & 0,80 \\
\hline 5 o más & 10 & 20,8 & 15 & 25,0 & & 0,67 \\
\hline Recién nacidos macrosómicos previos & 0 & - & 3 & 5,0 & 0,0021 & 0,00 \\
\hline Abortos recurrentes & 3 & 6,25 & 4 & 6,7 & 0,4522 & 0,75 \\
\hline Óbitos & 2 & 4,2 & 1 & 1,7 & 0,0771 & 2,00 \\
\hline Peso materno & & & & & 0,6230 & \\
\hline Normal & 5 & 10,4 & 2 & 3,3 & & 2,87 \\
\hline Sobrepeso & 4 & 8,3 & 3 & 5 & & 2,87 \\
\hline Obesidad & 22 & 45,8 & 24 & 40 & & 11,32 \\
\hline Obesidad severa & 17 & 35,4 & 31 & 51,7 & & 3,22 \\
\hline Aumento excesivo en el embarazo & 40 & 83,3 & 41 & 68,3 & 0,5689 & 0,98 \\
\hline Antecedentes personales & & & & & 0,0616 & \\
\hline Diabetes gestacional & 48 & 100 & 42 & 70 & & 1,14 \\
\hline Enfermedades tiroideas & 3 & 6,25 & 0 & - & & \\
\hline Hipertensión en el embarazo & 2 & 4,2 & 0 & - & & \\
\hline Antecedentes familiares & & & & & 0,6040 & \\
\hline Hipertensión arterial crónica & 34 & 100 & 0 & - & & \\
\hline Diabetes mellitus tipo 2 & 29 & 60,4 & 12 & 20 & & 2,42 \\
\hline Condiciones socioeconómicas & & & & & 0,1880 & \\
\hline Bajo nivel educativo & 9 & 18,8 & 0 & - & & \\
\hline Bajo ingreso económico & 48 & 100 & 36 & 60 & & 1,33 \\
\hline
\end{tabular}




\section{LUGO LEÓN ET AL.}

Tabla 7. Distribución según los factores de riesgo para complicaciones neonatales de la diabetes gestacional

\begin{tabular}{|c|c|c|c|c|c|c|}
\hline \multirow{3}{*}{ Factor de riesgo } & \multicolumn{4}{|c|}{ Neonatos de madres con diabetes gestacional } & \multirow{3}{*}{$\mathrm{p}$} & \multirow{3}{*}{$\begin{array}{l}\text { Riesgo } \\
\text { relativo }\end{array}$} \\
\hline & \multicolumn{2}{|c|}{ Complicados } & \multicolumn{2}{|c|}{ No complicados } & & \\
\hline & $\mathrm{n}=52$ & $\%$ & $\mathrm{n}=56$ & $\%$ & & \\
\hline Edad & & & & & 0,0001 & \\
\hline Inferior a 35 años & 22 & 42,3 & 3 & 5,3 & & 4,10 \\
\hline 35 años o más & 30 & 57,7 & 53 & 94,7 & & 7,34 \\
\hline Paridad & & & & & 0,0002 & \\
\hline Primigestas & 12 & 23,1 & 8 & 13,4 & & 1,63 \\
\hline 2 a 4 & 30 & 57,7 & 33 & 59,9 & & 4,60 \\
\hline 5 o más & 10 & 19,2 & 15 & 26,7 & & 1,25 \\
\hline Recién nacidos macrosómicos previos & 2 & 3,8 & 1 & 1,8 & 0,0738 & 2,00 \\
\hline Abortos recurrentes & 3 & 5,8 & 4 & 5,3 & 0,3319 & 0,75 \\
\hline Óbitos & 2 & 3,8 & 1 & 1,8 & 0,0738 & 2,00 \\
\hline Peso materno & & & & & 0,5980 & \\
\hline Normal & 4 & 7,7 & 3 & 5,4 & & \\
\hline Sobrepeso & 6 & 11,5 & 1 & 1,8 & & 1,472 \\
\hline Obesidad & 21 & 40,4 & 25 & 44,6 & & 8,422 \\
\hline Obesidad severa & 21 & 40,4 & 27 & 48,2 & & 2,455 \\
\hline Aumento excesivo de peso en el embarazo & 34 & 65,4 & 47 & 84 & 0,2523 & 2,72 \\
\hline \multicolumn{7}{|l|}{ Antecedentes personales } \\
\hline Diabetes gestacional & 52 & 100 & 38 & 67,9 & 0,000 & 5,45 \\
\hline Enfermedades tiroideas & 2 & 3,8 & 1 & 1,9 & 0,6078 & 1,831 \\
\hline Hipertensión en embarazos previos & 2 & 3,8 & 0 & - & 0,2295 & 5,594 \\
\hline Antecedentes familiares & & & & & 0,0019 & \\
\hline Hipertensión arterial crónica & 24 & 46,2 & 10 & 17,9 & & 3,807 \\
\hline Diabetes mellitus tipo 2 & 30 & 57,7 & 11 & 19,6 & & 5,363 \\
\hline Condiciones socioeconómicas & & & & & 0,0852 & \\
\hline Bajo nivel educativo & 7 & 13,5 & 2 & 3,6 & & 3,593 \\
\hline Bajo ingreso económico & 52 & 100 & 32 & 57,1 & & 3,15 \\
\hline
\end{tabular}

\section{DISCUSIÓN}

El impacto del diagnóstico de DG radica en que este trastorno tiene inmediatas consecuencias para el desarrollo del embarazo e implicaciones a largo plazo tanto para el recién nacido, como para la madre. Se estima que $6 \%-7 \%$ de los embarazos se complican con DM. El $90 \%$ de los casos representan mujeres con DG, mientras que $10 \%$ restante corresponden a DM tipo 1 y 2. El aumento de la prevalencia de DG 
se encuentra en gran asociación con el aumento de la obesidad y el sedentarismo (9).

En este estudio realizado en la población venezolana, se examinaron las historias clínicas de 108 pacientes con diagnóstico de DG y se compararon con los datos de las historias clínicas de 108 pacientes que no presentaban tal diagnóstico con el fin de establecer los principales factores de riesgo presentes en las pacientes para cursar o desarrollar complicaciones, tanto maternas como neonatales; en tal sentido se pudo evidenciar que la edad de las pacientes, tiempo de embarazo y paridad no tuvieron diferencias estadísticamente significativas entre los grupos, por lo tanto quedan desestimadas como factores de riesgo en esta población. Solo el $21 \%$ de las pacientes tenían niveles de instrucción que incluían el bachillerato y universitario completo, mientras que el $79 \%$ restante no ejercía ninguna profesión, lo cual implica un bajo nivel sociocultural que conlleva a la imposibilidad para mantener una dieta equilibrada durante la gestación que permita la disminución o prevención de los trastornos metabólicos y complicaciones perinatales, datos que concuerdan con Caiafa y cols. (10), quienes reportaron que las condiciones socioeconómicas, el bajo nivel educativo, el bajo ingreso económico y la inestabilidad del hogar, llevan a las pacientes a modificar sus estilos de vida, aumentando la ingesta de alimentos industrializados y azucares refinadas, aunado a hábitos de sedentarismo debido a mayor permanencia en el hogar; estas características como factores de riesgo potencian y condicionan la DG en las mujeres.

En cuanto al sobrepeso y la obesidad, se pudo observar que en ambos grupos hay una gran variación entre las pacientes, pero el grupo control manejó una mediana de IMC de $24 \mathrm{~kg} / \mathrm{m}^{2}$ mientras que el grupo experimental tiene una mediana de $30 \mathrm{~kg} / \mathrm{m}^{2}$. Al comparar ambos grupos se encontró asociación estadísticamente significativa, pudiendo estimar que un IMC mayor a $25 \mathrm{~kg} / \mathrm{m}^{2}$ se asoció a un riesgo 14 veces mayor para desarrollar DG, lo cual concuerda con lo observado en el Manual Venezolano de Diabetes Gestacional (5), en los estudios de Hod y cols. (11), en la publicación de Carreno y cols. (12), así como la de Caiafa y cols. (10), en los cuales el IMC materno mayor a $25 \mathrm{Kg} / \mathrm{m}^{2}$ es considerado factor importante en el desarrollo de diabetes durante la gestación.

En relación con la presencia de antecedentes familiares de diabetes como factor de riesgo, al comparar ambos grupos se encontraron diferencias estadísticamente significativas, estas pacientes tienen 2,8 veces más riesgo de desarrollar $\mathrm{DG}$, lo cual tiene relación con el estudio de Caiafa y cols. (10) debido al incremento que tiene en su casuística la presencia de antecedentes familiares de diabetes mellitus y su asociación con el desarrollo de DG. Si el antecedente personal es la DG, la paciente tendrá 130 veces más riesgo de desarrollar DG en un próximo embarazo, datos que coinciden con el estudio realizado en el servicio de endocrinología de la Maternidad Concepción Palacios en 2000, en el cual los factores de riesgo más importantes para desarrollar DG fueron la obesidad y los antecedentes personales de DG (13); también existe relación con lo reportado por el Manual Venezolano de Diabetes Gestacional (5), el boletín práctico 173 del American College of Obstetricians and Gynecologists (ACOG) (14) y el trabajo de Holmes y cols. (15), los cuales encontraron relación en el diagnóstico de $\mathrm{DG}$ en aquellas pacientes que tenían DG en embarazo anteriores.

En esta investigación, la ganancia de peso durante el embarazo fue evaluada, logrando precisar que el $75 \%$ de las pacientes del grupo experimental lograron un aumento mayor a $10 \mathrm{~kg}$ durante la gestación, posteriormente al ser comparados ambos grupos, se obtuvo significancia estadística, constatando que dichas pacientes tienen 4,2 veces más riesgo de desarrollar DG, datos que se correlacionan con Carreno y cols. (12), Alberico y cols. (16) y Hod y cols. (11), quienes encontraron asociación en la aparición del DG y el aumento de peso excesivo durante la gestación. 
Un grupo importante de las pacientes que tenían el diagnóstico de DG, se complicaron con hiperglicemias (43,5\%), dificultando el manejo, ameritando tratamiento farmacológico y aumentando la incidencia de comorbilidades asociadas como RPM (16\%) y THE (27\%). Esto guarda relación con Da Ros y cols. (17) quienes consideran que la DG es un factor de riesgo para el desarrollo de preeclampsia; estas complicaciones conllevaron a que $44,4 \%$ de las pacientes permanecieran más de 8 días en la institución.

Las complicaciones más frecuentes de los recién nacidos de madres con DG fueron la hipoglicemia, seguida de la macrosomía neonatal, motivo por el cual 10 de los recién nacidos presentaron apgar menor a 5 en el primer minuto y 49 recién nacidos, correspondiente al $45 \%$ ameritaron ingreso a UTIN, lo cual tiene relación con los estudios de Chu y cols. (18) quienes encontraron una puntuación de apgar igual o menor a 5 durante el primer minuto de vida en hijos de pacientes con DG; en el estudio de Alberico y cols. (16) y en el boletín práctico 137 del ACOG (14) se observó relación entre la DG y la macrosomía fetal.

Luego, como propósito fundamental de este estudio, se evaluaron los factores de riesgo presentes en aquellas pacientes con DG que se complicaron, por tanto, se evaluaron las diferencias entre las diabéticas gestacionales complicadas con THE, con hiperglicemia y con estancia hospitalaria prolongada, que fueron las complicaciones que se presentaron con diferencia estadística entre este grupo y las no diabéticas, es decir, que se asociaron significativamente con el hecho de tener diabetes gestacional.

Se observó que los THE se presentaron en 29 pacientes, 26,9\% de las diabéticas gestacionales, y solo en 16 de las no diabéticas, $14,8(\mathrm{p}=0,004)$. Las diabéticas complicadas con THE, en su mayoría, un $82 \%$, tenían más de 35 años y más de 2 embarazos, ninguna de estas pacientes tenía antecedente de $\mathrm{RN}$ macrosómicos pero si tenían DG en embarazos previos y antecedentes familiares de hipertensión y DM. Por el contrario, las diabéticas que no desarrollaron THE, eran más jóvenes ( $\mathrm{p}=0049$ ), en su mayoría, tenían entre 2 y 4 embarazos previos $(0,0012)$, tenían antecedentes de $\mathrm{RN}$ macrosómicos $(\mathrm{p}=0,0001)$ y tenían menor frecuencia de diabetes gestacional en embarazos anteriores $(p=0,0135)$. Si bien la DG no se encuentra dentro de las principales causas de mortalidad materna, las complicaciones tales como la preeclampsia están ligadas a las madres diabéticas $(3,8)$. Así, Caiafa y cols. (10), en Venezuela, encontraron entre las principales complicaciones maternas a la hipertensión arterial inducida por el embarazo. Sin embargo, en 2008, Da Ros y cols. (17) informaron que, a pesar de que muchos estudios consideran la DG como un factor de riesgo para la preeclampsia, lo que realmente ocurre es que existe una común causalidad para ambas enfermedades. De la presente investigación se infiere que el riesgo de que exista la comorbilidad (DG y THE) aumenta con la edad, la paridad, el antecedente personal de DG y los antecedentes familiares.

La hiperglicemia fue desarrollada por el 43,5 \%. El riesgo de desarrollarla fue 4 veces mayor en gestantes menores de 35 años lo que se correlaciona con el trabajo de Caiafa y cols. (10) quienes observaron que uno de los factores de riesgo para complicaciones era la edad materna superior a los 25 años. La presencia de hiperglicemia también se observó con más frecuencia cuando había antecedente de RN macrosómicos. Tal vez, la edad más joven y la presencia del antecedente de macrosomía, reflejen un menor control metabólico, el cual suele ser difícil de alcanzar en pacientes jóvenes que podrían no tomar conciencia real de la importancia del control dietético.

La mayor estancia hospitalaria se encontró en 48 mujeres diabéticas. Llama la atención que ninguna de las no diabéticas requirió estar hospitalizada más de 8 días. Los factores de riesgo evaluados no se relacionaron directamente con el tiempo de hospitalización, pero al asociarse THE e hiperglicemia, es fácil entender 
que las pacientes requieran cuidados particulares que ameriten mayor estancia hospitalaria.

Los factores de riesgo que se asociaron a complicaciones en los recién nacidos de madres con diabetes gestacional fueron ser menor de 35 años, lo cual aumenta el riesgo en más de 4 veces, primigesta, con antecedentes personales de DG y antecedentes familiares de DM e hipertensión arterial.

En conclusión, los principales factores de riesgo para desarrollar DG, fueron IMC mayor a $35 \mathrm{~kg} /$ $\mathrm{m} 2$, antecedentes personales de DG y enfermedades tiroideas, y antecedentes familiares de diabetes e hipertensión arterial crónica.

Las complicaciones maternas relacionadas con la DG fueron THE e hiperglicemia. Las complicaciones neonatales asociadas a DG fueron hipoglicemia, macrosomía, apgar menor a 5 en el primer minuto y mayor frecuencia de ingreso a UTIN.

La asociación entre diabetes gestacional y THE fue más frecuente en mayores de 35 años, con 2 o más embarazos previos y antecedentes familiares de hipertensión crónica y DM.

La asociación entre DG e hiperglicemia fue más frecuente en menores de 35 años.

Las complicaciones neonatales en pacientes con DG se asociaron a edad menor de 35 años, primigestas, antecedente de DG y antecedentes familiares de hipertensión crónica y DM.

\section{Sin conflictos de interés.}

\section{REFERENCIAS}

1. Bellart J, Pericot A, Vinagre I. Protocolo diabetes pregestacional [Internet]. Barcelona: Servei de Medicina Materno-fetal, Servei d'Endocrinologia i Nutrició, Unitat de Diabetis. Hospital Clínic de Barcelona; 2011 [consultado febrero 2017]. Disponible en: https://medicinafetalbarcelona.org/protocolos/ es/patologia-materna-obstetrica/diabetes $\% 20$ pregestacional.pdf

2. American Diabetes Association. 12. Management of Diabetes in Pregnancy. Diabetes Care. 2016; 39 Suppl 1:S94-8. doi: 10.2337/dc16-S015.

3. Goldenberg RL, McClure EM, Harrison MS, Miodovnik M. Diabetes during Pregnancy in Lowand Middle-Income Countries. Am J Perinatol. 2016; 33(13):1227-1235. doi: 10.1055/s-0036-1584152.

4. Erem C, Kuzu UB, Deger O, Can G. Prevalence of gestational diabetes mellitus and associated risk factors in Turkish women: the Trabzon GDM Study. Arch Med Sci. 2015; 11(4):724-735. doi: 10.5114/ aoms.2015.53291.

5. Brajkovich I, Febres Balestrini F, Camejo M, Palacios A; Sociedad Venezolana de Endocrinología y Metabolismo, Sociedad de Obstetricia y Ginecología de Venezuela y Sociedad Venezolana de Medicina Interna. Manual venezolano de diabetes gestacional. Rev Venez Endocrinol Metab [Internet]. 2016 [consultada febrero 2017]; 14(1): 56-90. Disponible en: https://www. svemonline.org/wp-content/uploads/2016/04/revistasvem-vol-14-1-2016.pdf

6. International Association of Diabetes and Pregnancy Study Groups Consensus Panel, Metzger BE, Gabbe SG, Persson B, Buchanan TA, Catalano PA, Damm P, et $a l$. International association of diabetes and pregnancy study groups recommendations on the diagnosis and classification of hyperglycemia in pregnancy. Diabetes Care. 2010; 33(3):676-682. doi: 10.2337/dc09-1848.

7. Coustan DR, Lowe LP, Metzger BE, Dyer AR; International Association of Diabetes and Pregnancy Study Groups. The Hyperglycemia and Adverse Pregnancy Outcome (HAPO) study: paving the way for new diagnostic criteria for gestational diabetes mellitus. Am J Obstet Gynecol. 2010; 202(6):654.e1-6. doi: 10.1016/j.ajog.2010.04.006.

8. Goldenberg RL, McClure EM. Maternal, fetal and neonatal mortality: lessons learned from historical changes in high income countries and their potential application to low-income countries. Matern Health Neonatol Perinatol. 2015; 1:3. doi: 10.1186/s40748014-0004-z.

9. Scucces M. Diabetes y embarazo. Rev Obstet Ginecol Venez [Internet]. 2011 [consultado febrero de 2017]; 71(1): 3-12. Disponible en: http://www.sogvzla. org.ve/sogvzla $20186 / \mathrm{cms} / \mathrm{svcobtenerpdfrevista.}$ php?id $=0000000009 \&$ tipo $=$ normal\&fila $=3$ 
10. Caiafa J, Rodríguez Z, Carrizales ME, Herrera M. Caracterización epidemiológica de la diabetes gestacional. Estado Aragua. Año 2000-2007. Comunidad y Salud [Internet]. 2010 [consultado febrero de 2017]; 8 (2): 14-24. Disponible en: http://ve.scielo. org/scielo.php?script $=$ sci_arttext $\&$ pid $=S 1690$ 32932010000200003\&lng=es

11. Hod M, Kapur A, Sacks DA, Hadar E, Agarwal M, Di Renzo GC, et al. The International Federation of Gynecology and Obstetrics (FIGO) Initiative on gestational diabetes mellitus: A pragmatic guide for diagnosis, management, and care. Int J Gynaecol Obstet. 2015; 131 Suppl 3:S173-S211. doi: 10.1016/ S0020-7292(15)30033-3.

12. Carreno CA, Clifton RG, Hauth JC, Myatt L, Roberts JM, Spong CY, et al.; Eunice Kennedy Shriver National Institute of Child Health and Human Development (NICHD) Maternal-Fetal Medicine Units (MFMU) Network. Excessive early gestational weight gain and risk of gestational diabetes mellitus in nulliparous women. Obstet Gynecol. 2012; 119(6):1227-1233. doi: 10.1097/AOG.0b013e318256cfla.

13. Febres F, Palacios A, Colán J, Arias E. Nueva pesquisa de consenso de la diabetes gestacional, en función del futuro inmediato y a largo plazo del recién nacido. Rev Obstet Ginecol Venez [Internet]. 2014 [consultado febrero 2017]; 74 (2): 138-142. Disponible en: http:/www.sogvzla. org.ve/sogvzla20186/cms/svcobtenerpdfrevista. php?id $=0000000071 \&$ tipo $=$ normal $\&$ fila $=9$

14. Practice Bulletin No. 137: Gestational diabetes mellitus. Obstet Gynecol. 2013; 122(2 Pt 1):406-416. doi: 10.1097/01.AOG.0000433006.09219.f1.
15. Holmes HJ, Lo JY, McIntire DD, Casey BM. Prediction of diabetes recurrence in women with class A1 (diettreated) gestational diabetes. Am J Perinatol. 2010; 27(1):47-52. doi: 10.1055/s-0029-1241733.

16. Alberico S, Montico M, Barresi V, Monasta L, Businelli C, Soini V, et al.; Multicentre Study Group on Mode of Delivery in Friuli Venezia Giulia. The role of gestational diabetes, pre-pregnancy body mass index and gestational weight gain on the risk of newborn macrosomia: results from a prospective multicentre study. BMC Pregnancy Childbirth. 2014; 14:23. doi: 10.1186/1471-2393-14-23.

17. Wendland EM, Duncan BB, Belizán JM, Vigo A, Schmidt MI. Gestational diabetes and pre-eclampsia: common antecedents? Arq Bras Endocrinol Metabol. 2008 ;52(6):975-984. doi: 10.1590/s000427302008000600008.

18. Chu SY, Callaghan WM, Kim SY, Schmid CH, Lau $\mathrm{J}$, England LJ, et al. Maternal obesity and risk of gestational diabetes mellitus. Diabetes Care. 2007; 30(8):2070-2076. doi: 10.2337/dc06-2559a.

19. Corrigan N, Brazil DP, McAuliffe F. Fetal cardiac effects of maternal hyperglycemia during pregnancy. Birth Defects Res A Clin Mol Teratol. 2009; 85(6):523530. doi: 10.1002/bdra.20567.

Recibido 17 de junio de 2021 Aprobado 14 de septiembre de 2021 